\title{
ArcheoSciences
}

Revue d'archéométrie

\section{Les activités métallurgiques dans les établissements ruraux enclos du second âge du Fer en Basse-Normandie : l'exemple du site des « Pleines » à Orval (Manche)}

Metallurgical activities in enclosed rural settlements during the 2nd Iron Age in Normandy: The example of the site of Orval, Les Pleines (Manche)

Nolwenn Zaour, Hubert Lepaumier, Marion Berranger et Philippe Fluzin

\section{OpenEdition}

Journals

Édition électronique

URL : https://journals.openedition.org/archeosciences/4252

DOI : $10.4000 /$ archeosciences. 4252

ISBN : 978-2-7535-3691-3

ISSN : 2104-3728

Éditeur

Presses universitaires de Rennes

Édition imprimée

Date de publication : 30 novembre 2014

Pagination : 165-181

ISBN : 978-2-7535-3689-0

ISSN : 1960-1360

Référence électronique

Nolwenn Zaour, Hubert Lepaumier, Marion Berranger et Philippe Fluzin, «Les activités métallurgiques dans les établissements ruraux enclos du second âge du Fer en Basse-Normandie : l'exemple du site des «Pleines » à Orval (Manche) », ArcheoSciences [En ligne], 38 | 2014, mis en ligne le 30 novembre 2016, consulté le 01 février 2022. URL : http://journals.openedition.org/archeosciences/4252 ; DOI : https://doi.org/10.4000/archeosciences.4252 


\title{
Les activités métallurgiques dans les établissements ruraux enclos du second âge du Fer en Basse-Normandie : l'exemple du site des « Pleines » à Orval (Manche)
}

\author{
Metallurgical Activities in Enclosed Rural Settlements During the $2^{\text {nd }}$ Iron Age \\ in Normandy: The Example of the Site of Orval, Les Pleines (Manche)
}

\author{
Nolwenn ZaOUR ${ }^{\mathrm{a}}$, Hubert LePaUmieR ${ }^{\mathrm{b}}$, Marion BerRanger ${ }^{\mathrm{c}}$ \\ et Philippe FLuZIN ${ }^{c}$
}

\begin{abstract}
Résumé : Identifiés dès le diagnostic archéologique, les déchets métallurgiques du site des Pleines à Orval (Manche) ont fait l'objet d'un protocole d'étude spécifique durant la fouille. La répartition spatiale, en particulier des battitures, montre la présence d'au moins un atelier associé à la première phase d'occupation du site (La Tène ancienne/La Tène moyenne). L'analyse métallographique des culots de forge permet de préciser les types d'activités pratiquées et la nature des productions. Nous proposons donc une étude de synthèse (archéologique et archéométrique) des principaux résultats sur ce site en regard du contexte régional. La présence de déchets polymétallurgiques (fer et alliage cuivreux) sur les occupations encloses du second âge du Fer n'est d'ailleurs pas rare. Le recensement des indices liés à ces activités montre que plus de $60 \%$ des établissements bas-normands, fouillés depuis 1991, devaient être dotés d'une forge. Ce taux illustre une pratique courante et largement répandue des travaux de post réduction sur ces habitats ruraux gaulois, et en fait une des activités artisanales les mieux représentées.
\end{abstract}

\begin{abstract}
The metallurgical waste products of the Orval "Les Pleines" site in la Manche which were evidenced during archaeological trial trenching have been studied with a specific protocol developed during the excavation. The distribution, of specific magnetic elements, shows the presence of at least one workshop associated with the first phase of occupation of the site (early La Tènelmiddle La Tène). The metallographic analysis of the forging slag-cakes makes it possible to specify the types of activities practiced and the nature of the productions. We thus propose a synthetic study both archaeological and archeometric of the main results in regard of the local context. The presence of poly-metallic waste (iron and copper alloy) from the second Iron Age enclosure settlement is indeed not rare. The inventory of the remains associated with these activities shows that over $60 \%$ of the Norman settlements excavated since 1991 most probably comprised a forge. This rate demonstrates how common the practise of poste réduction was in these Gallic rural settlements, making forging actually one of the best represented craft activities
\end{abstract}

Mots-clés : Âge du Fer, Forge, Métallurgie, Fer, Alliage cuivreux, Normandie.

Keywords: Iron Age, Forging activity, Metallurgy, Iron, Copper Alloy, Normandy.

\footnotetext{
a INRAP Grand Ouest, LMC UMR 5060 CNRS IRAMAT

b INRAP Grand Ouest, UMR 6566 CNRS CREAAH

${ }^{\mathrm{c}}$ LMC UMR 5060 CNRS IRAMAT
} 
Paradoxalement, s'il est une activité assez mal documentée sur les établissements de l'âge du fer, c'est bien celle afférente au travail même du Fer. Cette carence semble pourtant être davantage liée au caractère peu spectaculaire et peu démonstratif des témoins que cette activité a le plus souvent engendrés. En l'absence quasi systématique de structure ou de mobilier spécifique lié à cet artisanat (fours, pinces, outils), il ne subsiste plus que quelques déchets, peu caractéristiques de prime abord et qui ne font le plus souvent l'objet que d'une simple mention, sans description ni détermination précise. Parfois totalement délaissés, rarement considérés à leur juste valeur, les déchets issus des diverses activités métallurgiques sont le plus souvent regroupés sous le vocable de scorie.

La mise en évidence de scories lors de la phase de diagnostic sur le site des " Pleines " à Orval (Manche) a permis de mettre en place dès le début de l'opération de fouille un protocole d'étude spécifique visant à mieux quantifier et qualifier les activités métallurgiques qui s'y sont déroulées. Ces données ont été complétées par des analyses métallographiques réalisées au Laboratoire métallurgies et cultures UMR 5060 - IRAMAT - CNRS à l'Université de Technologie Belfort Montbéliard. Au-delà de cet exemple, nous avons tenté d'évaluer l'importance de ces pratiques sur les sites ruraux enclos d'époque gauloise de Basse-Normandie. À cette fin, nous avons dépouillé les Bilans Scientifiques Régionaux de Basse-Normandie depuis 1991, année de leur mise en place jusqu'en 2011, année du dernier bulletin paru au moment de rédiger ces lignes. Cette base de travail a permis d'établir la liste des sites fouillés au cours de cette période de référence. Dans la mesure du possible, nous nous sommes reportés aux rapports de fouille et autres documents finaux de synthèse afin de vérifier plus en détail le signalement éventuel de la présence d'activités métallurgiques. Enfin, pour les quelques sites publiés, il a été vérifié qu’aucun élément nouveau ne venait compléter les données déjà acquises.

\section{Le Site des « Pleines » à Orval}

La fouille du site d'Orval s'est inscrite dans le cadre des travaux liés au contournement ouest de la ville de Coutances. Les travaux financés par le Conseil Général de la Manche, maître d'œuvre du tracé routier, ont porté sur une surface de près de 2 ha. La fouille du site supervisée par David Giazzon (INRAP) a permis de restituer trois phases d'occupations domestiques distinctes qui s'échelonnent entre le début du $\mathrm{III}^{\mathrm{e}}$ siècle avant notre ère et le début du $\mathrm{I}^{\mathrm{er}}$ siècle de notre ère.

La découverte de scories lors du diagnostic nous avait alertés quant à la probable présence d'activités de forge sur le site. Nous avions donc décidé au moment de la phase de terrain de surveiller particulièrement l'apparition des vestiges liés à cet artisanat.

Une concentration de scories rencontrée dans l'un des premiers sondages réalisés sur le site a rapidement couronné cette attention. Ces nouveaux indices nous ont alors incités à réaliser et à tester quelques prélèvements de sédiments afin de voir si des éléments magnétiques millimétriques comme des battitures ne s'y trouvaient pas également. Ces battitures sont des oxydes de fer de très petites tailles qui se forment par oxydation au contact de l'air sur la masse de métal chauffée. Elles se détachent du bloc de fer lorsque celui-ci est martelé par le forgeron. Elles se distinguent généralement en trois catégories. Elles sont dites boursoufflées lorsque la surface de la battiture présente des irrégularités; planes lorsqu'elles apparaissent sous forme de lamelles, de feuilles ou de paillettes. Elles peuvent encore adopter la forme d'une bille. Elles sont caractéristiques des activités de forgeage : martelage et soudure (Berranger, 2013; Leblanc, 2001). Par ailleurs, en raison de leur petite taille, ces déchets ne font généralement pas l'objet d'une gestion raisonnée et leur présence en grande quantité signe souvent la proximité de l'atelier. Dans l'absolu, lorsque les sols sont conservés (ce qui n'était malheureusement pas le cas ici), les concentrations de battitures peuvent être à même de préciser l'emplacement de l'enclume au sein de l'atelier. En raison des difficultés de lecture permettant de différencier les structures archéologiques ponctuelles, de types fosses ou trous de poteau, des structures "naturelles ", de type chablis ou terriers, nous avons concentré nos efforts sur les nombreux fossés présents sur le site. Certes, ce choix présentait l'inconvénient majeur de ne pas pouvoir localiser précisément le ou les ateliers mais il devait être à même d'en estimer le nombre et surtout d'en établir la ou les spécificités.

Dans le sondage du fossé 171 qui a servi de zone test, un sac de sédiment de 800 à $900 \mathrm{~g}$ a été prélevé tous les $10 \mathrm{~cm}$. Les tests mis en ouvre afin de vérifier la présence de micro déchets telles que les battitures se sont révélés positifs dans toutes les strates du fossé. Nous avons donc établi un protocole de prélèvement systématique d'un sac de sédiment de $500 \mathrm{~g}$ à $1000 \mathrm{~g}$ à $20 \mathrm{~cm}$ de profondeur dans tous les sondages de tous les fossés du site (sondage de $2 \mathrm{~m}$ ).

Ce sont en tout 182 prélèvements provenant de 14 structures différentes qui ont ainsi été tamisées.

Enfin, il convient d'ores et déjà de préciser que si la majorité des vestiges archéologiques métallurgiques découverts sur le site concerne le travail du fer, une partie est également à mettre en relation avec le travail des alliages base cuivre. 


\section{$1^{\text {re }}$ phase : l'occupation de la fin de La Tène ancienne}

Dans son premier état, l'établissement présente un enclos central trapézoïdal d'un peu moins de $2000 \mathrm{~m}^{2}$ précédé par un parcellaire et un réseau viaire. Cette séquence est marquée par la présence d'une tombe à char installée dans l'un des angles du chemin de desserte de l'enclos (Lepaumier $e t$ al. 2011a). L'important mobilier métallique mis au jour dans cette tombe, tout comme les éléments céramiques rencontrés dans le comblement des fosses et fossés suggèrent une datation au cours de la première moitié du $\mathrm{III}^{\mathrm{e}}$ siècle avant notre ère, vers la fin de La Tène ancienne et la transition avec La Tène moyenne. Cette première phase d'occupation est celle qui a livré le plus de matériel métallurgique concernant aussi bien le travail du fer que celui des alliages base cuivre. Ainsi $77 \%$ des déchets métallurgiques du site se retrouvent dans cette première phase. Au total ce sont quelques 65 culots de forge et fragments de scories de forge, 19 fragments de parois scoriacées, 5 empreintes d'évents ainsi que 4 creusets qui ont pu être attribués à cette phase. L'ensemble de ces rejets totalise une masse de $5,4 \mathrm{~kg}$.

Deux zones de concentration de déchets sidérurgiques ont été repérées (fig. 1a). La première (zone 1) se situe à l'angle des fossés 131 et 150 et la seconde (zone 2), en face, à l'angle des fossés 150 et 171 . La répartition spatiale des éléments magnétiques confirme également ce plan avec de très forte concentration de battitures au niveau de ces deux angles (fig. 1b). Ces deux zones sont en fait assez comparables quant à la nature des déchets qu'elles ont livrés. Nous y avons trouvé des culots de forge, des scories informes, des éléments de foyer et un grand nombre de battitures. La première différence que nous pouvons cependant noter est une plus forte présence de culots dans la zone 1, répartition spatiale qui va à l'inverse de celle observée pour les battitures dont les concentrations sont beaucoup plus fortes dans la zone 2. Mais la distinction principale entre ces deux aires se fait en réalité au niveau des déchets liés au travail des alliages base cuivre. En effet, la quasi-totalité de ces derniers provient de la zone 1. Seul un petit fragment de creuset et deux gouttes de métal cuivreux proviennent de l'angle opposé, en zone 2.

Nous pourrions alors supposer que nous avons découvert deux ateliers distincts situés aux angles nord de l'enclos dont l'un, celui de la zone 1, aurait peut-être été plus spécialisé dans le travail des alliages base cuivre. L'idée est séduisante. Mais nous sommes également obligés de constater que la présence de ces déchets se superpose à celle des découvertes céramiques (fig. 1c). En effet, nous ne trouvons les résidus métallurgiques qu'en parallèle des zones à fortes concentra- tions de rejets céramiques. La conclusion que nous tirons de ces observations est que nous nous trouvons sans doute davantage dans des zones de rejets préférentiels de l'habitat plutôt que dans des espaces réservés aux ateliers sidérurgiques ou proches de ces derniers.

En dépit de cette constatation, l'exploitation des battitures, toutes phases confondues, nous permet de compléter le tableau.

Ainsi, il faut noter la présence de battitures dans des structures postérieures recoupant l'angle nord-est de l'enclos (zone 1). Mis à part les sondages de cette portion de fossé et un autre à son extrémité nord-est, il n'y a nulle part ailleurs dans ce fossé 13, de battitures. De plus, dans ce secteur que le fossé du Haut Empire traverse, aucune scorie susceptible de trahir la présence d'une forge n'est associée à cette dernière occupation. L'hypothèse la plus probable pour expliquer la présence de battitures dans le fossé 13 consiste donc à envisager que le creusement de ce dernier a perforé des structures ou des niveaux de sols liés à la forge de La Tène ancienne.

Pour l'autre zone (zone 2), on constate la présence de battitures dans toutes les strates du remplissage du fossé 171 . Mais les principales concentrations ont été rencontrées dans l'une des unités stratigraphiques qui semble marquer un recreusement du fossé. Celui-ci pourrait être lié à l'installation d'une palissade sur la façade d'accès à l'enclos. Ici, on se trouverait donc davantage face à une utilisation de remblais contenant, entre autres choses, des déchets métallurgiques. Ces remblais destinés à caler une palissade ne permettent donc pas de confirmer la proximité d'une forge.

\section{$2^{\text {de }}$ phase : une occupation de la La Tène finale}

Beaucoup plus discrète que les deux autres phases du site, l'occupation de la fin de l'âge du Fer se limite à quelques fossés peu profonds et quelques fosses. Cette discrétion est sans doute partiellement imputable au fait qu'une partie des structures nous est masquée par l'ultime occupation domestique. Il est probable qu'à l'origine l'habitat s'articulait autour d'un vaste enclos quadrangulaire à l'intérieur duquel ont été reconnues deux structures profondes, de type souterrain, et autour duquel se développait un parcellaire. D'après le mobilier céramique, ces structures paraissent rebouchées au cours du Ir siècle avant notre ère.

Tout aussi discrets que les structures, les déchets métallurgiques sont apparus dans cette phase en faible quantité ( $6 \%$ du nombre total des rejets métallurgiques) et très peu variés, à la différence de la première phase. Nous pouvons noter la présence d'un élément (inv. 076-004-001) de paroi scoriacée provenant de la métallurgie des alliages base cuivre. 

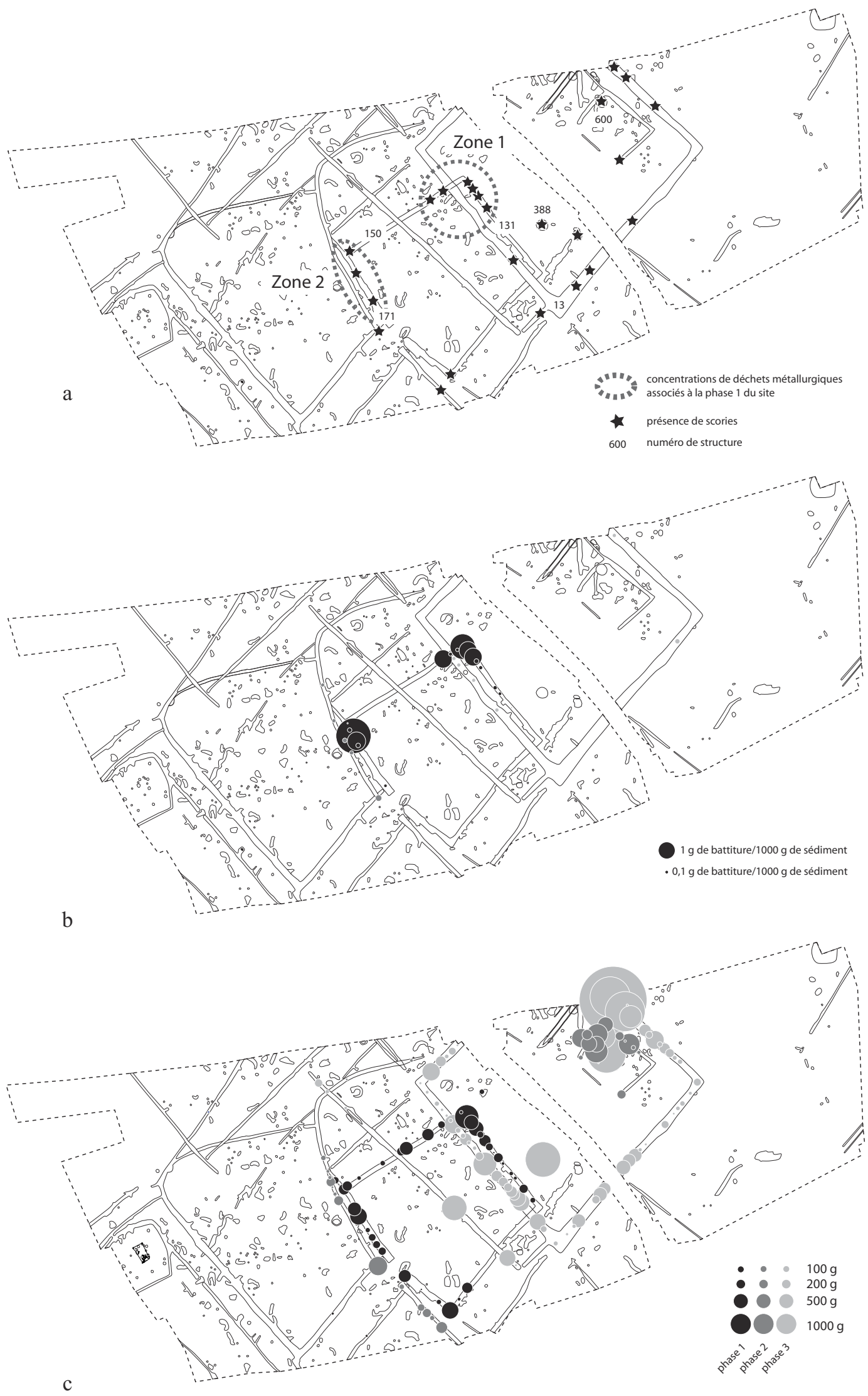

Figure $1:$ a. Orval "Les Pleines ", plan de répartition des déchets métallurgiques; b. Orval «Les Pleines ", plan de répartition des battitures; c. Orval "Les Pleines ", plan de répartition des rejets céramiques (noir : phase 1 ; gris foncé; phase 2 ; gris clair : phase 3 ). Figure 1: a. Orval "Les Pleines", distribution of the metallurgical waste; 1b. Orval "Les Pleines", distribution of hammerscales; 1c. Orval "Les Pleines", distribution of ceramics (black: phase 1; dark gray; phase 2; light grey: phase 3). 
Ce dernier a été découvert dans une structure située à l'est $\mathrm{du}$ site, alors que tous les autres déchets proviennent d'un fossé situé à l'opposé. La faible quantité de ces résidus rend toute étude spatiale difficile. Nous pouvons tout de même remarquer que leur diffusion concorde ici encore avec les zones de rejets céramiques.

\section{$3^{\mathrm{e}}$ phase : l'occupation du haut-Empire}

Cette troisième et dernière phase d'occupation s'organise autour d'un enclos quadrangulaire de $3600 \mathrm{~m}^{2}$ délimité par un fossé aux dimensions assez importantes. Parmi les structures singulières associées à cet épisode on peut noter tout particulièrement la présence d'une voirie et surtout de deux puits dont l'un est toujours en eau. Le mobilier métallique (deux fibules en particulier) et céramique permettent d'envisager un comblement des structures au cours du $\mathrm{I}^{\mathrm{er}}$ siècle de notre ère.

Cette phase regroupe $17 \%$ des déchets métallurgiques recueillis sur le site. À la différence des deux premières phases tous les déchets sont issus de la métallurgie du fer. Il n'y a aucune trace du travail des alliages base cuivre.

Seules trois structures (fossés et puits) sont concernées par la présence de ce matériel sans pour autant qu'une répartition spatiale organisée apparaisse. Nous pouvons relever que la présence de ces déchets coïncide également avec les zones à fortes concentrations de rejets céramiques. La majorité des déchets de cette phase proviennent des fossés de délimitations de l'enclos (fossé 13). Tous les autres proviennent des puits 388 et 600 .

\section{Une source d'informations complémentaires : les analyses métallographiques}

Si la présence de culots et de creusets montre que des activités métallurgiques ont été pratiquées sur le site, l'approche archéométrique réalisée a, quant à elle, pour but de mieux les cerner. Ces études n'ont pas pu être menées sur les déchets du travail des alliages base cuivre mais se sont concentrées sur les déchets sidérurgiques.

L'ensemble du mobilier métallurgique, qui compte 127 déchets pour un poids total de 7,4 kg, a donc été traité. Ils ont tous été identifiés et triés par catégories (parois de foyer, éléments de ventilation, culots de forge, fragments de scories de forge, creusets, déchets d'alliage base cuivre).

Les déchets reconnus sur le site ont donc permis de qualifier les travaux pratiqués. En effet, l'essentiel du corpus est constitué de culots de forge et de fragments de scories de forge. Les culots complets représentent à eux seuls $68 \%$ du poids total des déchets : 28 culots pour un poids total de
$5 \mathrm{~kg}$ dont 19 pour un poids de 3,4 $\mathrm{kg}$ pour la phase La Tène ancienne. Le poids de ces culots varie entre 28 gr et 458 gr et la moyenne est de $180 \mathrm{gr}$. Les dimensions, quant à elles, varient entre $3,9 \mathrm{~cm}$ à $11,3 \mathrm{~cm}$ pour l'axe de ventilation et $3,2 \mathrm{~cm}$ à $10,6 \mathrm{~cm}$ pour l'axe perpendiculaire. Ces scories ne peuvent avoir été formées qu'au cours d'un travail de forge et il est assuré, à Orval, qu'aucune activité de réduction ne s'est déroulée sur ou à proximité immédiate du site.

Les analyses se sont concentrées sur les culots de forge. Ceux-ci ont été classés en fonction de leur morphologie et de leur type et l'analyse a porté sur une sélection de neuf d'entre eux (tab. 1) en suivant le protocole d'étude mis au point par Philippe Fluzin (Mangin et al., 2000, p. 194-195). L'étude métallographique qui a pour but de déterminer la nature des matériaux et d'interpréter leurs conditions de formation a permis de caractériser plus finement le travail réalisé par les forgerons gaulois d'Orval.

Le corpus de culots complet a été divisé en trois groupes en fonction de critères morphologiques d'après le protocole mis au point par Vincent Serneels (Serneels 1993). Les Scories Grises Denses (SGD) concernent 21 culots dont 17 culots se retrouvent dans le groupe SGD1 et 4 culots dans le groupe SGD2. Les 7 culots restants ont été classés dans le groupe des Scories Argilo-Sableuses (SAS).

SGD1 : les culots de ce groupe, qui sont aussi les plus nombreux, ont une densité moyenne à forte. La forme est généralement ovalaire. La surface est couverte d'une couche d'argilo-sableux et la couleur oscille entre rouille et violette. Les culots présentent un aspect irrégulier agrémenté de quelques boursouflures et de zones lisses. Les réactions magnétiques peuvent être fortes ou absentes.

SGD2 : les culots de ce groupe sont massifs et de densité moyenne à forte. La forme est assez circulaire et la coupe plano-convexe. L'aspect de la surface est irrégulier. Ce type de culot peut être magnétique ou non.

SAS : les culots de ce groupe ont une densité légère. Ils sont formés d'argilo-sableux. Ils sont informes, à l'aspect irrégulier et ne présentent pas de réaction magnétique.

Un culot découvert lors de la phase de décapage du site qui n'a donc pu être attribué à l'une ou l'autre des phases, a été retenu car son identification d'un seul point de vue macroscopique restait incertaine. Ce culot incomplet se classe parmi les SGD. Deux autres culots proviennent de la phase la plus récente du site datée du Haut Empire. L'un d'eux est de type SGD1 et l'autre de type SGD2. La seconde phase du site, datée de La Tène finale, étant bien plus discrète, aucun échantillon n'a été sélectionné pour cette étude. L'essentiel de l'analyse métallographique a donc porté sur les culots de la phase la plus ancienne datée de la fin de La Tène ancienne avec une sélection de six exemplaires. Cinq d'entre 


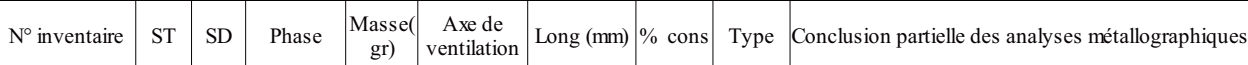

\begin{tabular}{|c|c|c|c|c|c|c|c|c|c|}
\hline \multirow[t]{2}{*}{$131-024-024$} & \multirow[t]{2}{*}{131} & \multirow[t]{2}{*}{24} & \multirow[t]{2}{*}{1} & \multirow[t]{2}{*}{273} & \multirow[t]{2}{*}{ oui } & \multirow[t]{2}{*}{$103 \times 87 \times 34$} & \multirow[t]{2}{*}{100} & \multirow[t]{2}{*}{ SGD1 } & $\begin{array}{l}\text { Ce culot présente une stratification. La partie inférieure, est constituée d'une } \\
\text { matrice base silice contenant des lattes de fayalites. On note une superposition de } \\
\text { différentes formes de cristallisation de la fayalite avec des lattes plus ou moins } \\
\text { épaisses et la présence de dendrites de wustite éparses. Cela reflete le cyclage } \\
\text { thermique caractéristique d'une opération de forge. Ces lattes s'allongent et } \\
\text { s'affinent vers le haut du culot. Ceci indique laapplication de températures élevées } \\
\text { suivie d'un refroidissement rapide. En partie supérieure de la hercynite/ magnétite } \\
\text { est présente, révélant peut être la présence d'ajouts siliceux. Dans la zone basse } \\
\text { quelques battitures à tendance globulaire ainsi que circulaires sont visibles. Une } \\
\text { très grande et épaisse battiture } 4 \text { (4,8 mm d'épaisseur) à tendance plane est présente } \\
\text { sur le fond du culot. Cette battiture marque le travail de forge d'un objet } \\
\text { volumineux }\end{array}$ \\
\hline & & & & & & & & & $\begin{array}{l}\text { II semble que le travail de forge ait été réalisé dans le cadre d'une bonne maîtrise } \\
\text { de l'oxydation à chaud. En effet, les pertes en oxydes (wüstite et en métal) sont } \\
\text { très faibles malgré l'augmentation des températures et l'hétérogénéité thermique } \\
\text { perçues. }\end{array}$ \\
\hline \multirow[t]{2}{*}{$131-024-025$} & \multirow[t]{2}{*}{131} & \multirow[t]{2}{*}{24} & \multirow[t]{2}{*}{1} & \multirow[t]{2}{*}{347} & \multirow[t]{2}{*}{ oui } & \multirow[t]{2}{*}{$99 \times 91 \times 37$} & \multirow[t]{2}{*}{100} & \multirow[t]{2}{*}{ SGD1 } & $\begin{array}{l}\text { Il s'agit d'un culot composite constitué de deux culots de forge dont les matrices } \\
\text { base silice contiennent de la fayalite à longues et fines lattes. Le culot inférieur est } \\
\text { caractérisé par la présence éparses de dendrites de wüstite, indiquant une faible } \\
\text { oxydation à chaud. Le second culot est de structure comparable. Toutefois, ici les } \\
\text { dendrites de wüs tite semblent provenir de la dissolution de battitures plus ou } \\
\text { moirs épaisses. }\end{array}$ \\
\hline & & & & & & & & & $\begin{array}{l}\text { Le travail de forge semble avoir été réalisé dans le cadre d'une bonne mâtrise de } \\
\text { l'oxydation à chaud. L'épaisseur des battitures, proportionnelle à la chauffe et aux } \\
\text { impacts mécaniques indiqueraient la forge de produits volumineux. }\end{array}$ \\
\hline \multirow[t]{2}{*}{$131-024-029$} & \multirow[t]{2}{*}{131} & \multirow[t]{2}{*}{24} & \multirow[t]{2}{*}{1} & \multirow[t]{2}{*}{118} & \multirow[t]{2}{*}{ oui } & \multirow[t]{2}{*}{$75 \times 78 \times 25$} & \multirow[t]{2}{*}{100} & \multirow[t]{2}{*}{ SGD1 } & $\begin{array}{l}\text { La matrice est présente sous forme de petits ilôts. Celle-ci est très riche en silice et } \\
\text { contient de la fayalite à fines lattes et des dendrites de wüstites éparses. Si lon } \\
\text { notre la présence de quelques billes de métal et de petits globules métalliques, il y } \\
\text { a aussi des fragments d'acier corrodé dont un de taille conséquente }(16 x 6 \mathrm{~mm}) \\
\text { dont la teneur en carbone pourrait être de } 0,7 \text { à } 0,8 \% \text {. On observe également } \\
\text { quelques battitures en cours de dissolution }\end{array}$ \\
\hline & & & & & & & & & $\begin{array}{l}\text { Ce culot semble avoir été constitué dans le cadre d'une faible oxydation à chaud en } \\
\text { lien avec des ajouts siliceux. La présence de fragments de métal corrodé dont un } \\
\text { fragment d'acier et de billes indiquent le travail de lacier. De même le gros fragment } \\
\text { de métal (16x6mm) marque le travail d'un objet de taille importante. }\end{array}$ \\
\hline \multirow[t]{2}{*}{$150-100-001$} & \multirow[t]{2}{*}{150} & \multirow[t]{2}{*}{100} & \multirow[t]{2}{*}{1} & \multirow[t]{2}{*}{76} & \multirow[t]{2}{*}{ oui } & \multirow[t]{2}{*}{$47 \times 80 \times 80$} & \multirow[t]{2}{*}{100} & \multirow[t]{2}{*}{ SAS } & $\begin{array}{l}\text { La matrice est majoritairement siliceuse. La fayalite présente différentes formes de } \\
\text { cristallisation, caratéristiques d'un cyclage thermique. Aucune perte en oxyde } \\
\text { (wüstite) n'a été observée. On note la présence de hercynite sans doute liée à une } \\
\text { contamination en aluminium provenant de la paroi du foyer conservée dans le } \\
\text { culot. }\end{array}$ \\
\hline & & & & & & & & & $\begin{array}{l}\text { L'hypothèse la plus vraisemblable est que ce culot se soit formé par l'altération des } \\
\text { parois du foyer. }\end{array}$ \\
\hline 171-006-001 & 171 & 6 & 1 & 255 & oui & $80 \times 70 \times 28$ & 100 & SGD1 & $\begin{array}{l}\text { La matrice base silice contient des lattes de fayalites présentes sous différentes } \\
\text { formes de cristallisation, ce qui est caractéristique d'un cyclage thermique. Il faut } \\
\text { également noter la présence de dendrites de wüstite éparses et ainsi que de } \\
\text { battitiures. Celles-ci peuvent être globulaires à tendances planes, biens } \\
\text { individualisées, et de tailles variées. }\end{array}$ \\
\hline & & & & & & & & & $\begin{array}{l}\text { Ce culot s'est constitué dans le cadre d'une oxydation à chaud bien mâtrtisée. On } \\
\text { observe ainsi la présence de battitures volumineuses, sans aucune perte en métal. } \\
\text { Le travail qui a engendré ce culot correspond sans doute à la forge d'objets } \\
\text { volumineux. }\end{array}$ \\
\hline $171-007-008$ & 171 & 7 & 1 & 190 & oui & $88 \times 73 \times 41$ & 95 & SGD1 & $\begin{array}{l}\text { La matrice base silice contient des lattes de fayalite ainsi que de mini-dendrites de } \\
\text { wüstite éparses et quelques petits globules de métal. } \\
\text { Dans la partie supérieure, les lattes de fayalite sont très longues et très fines } \\
\text { (températures élevées et refroidissement rapide) alors que la partie inférieure se } \\
\text { caractérise par une superposition de différentes formes de cristallisation, } \\
\text { caractéristique du cyclage thermique observé en forge. } \\
\begin{array}{l}\text { Le travail de forge semble avoir été effectué dans le cadre d'une bonne mấtrise des } \\
\text { conditions d'oxydation à chaud, malgré l'augmentation des températures. }\end{array} \\
\end{array}$ \\
\hline & & & 3 & & & & & & $\begin{array}{l}\text { La matrice base silice est saturée de globules de wustite, provennant de la } \\
\text { dissolution de battitures. La stratification de ces pertes est visible à l'œeil nu. } \\
\text { L'absence de métal est à noter. }\end{array}$ \\
\hline $013-007-003$ & 13 & 7 & 3 & 507 & our & $105 \times 92 \times 43$ & 80 & SGDI & $\begin{array}{l}\text { Ce culot a été constitué dans le cadre d'une forte oxydation à chaud. Le travail } \\
\text { devait donc concerner des produits suffisamment volumineux pour supporter de } \\
\text { telles pertes en oxydes. }\end{array}$ \\
\hline $600-000-001$ & 600 & 0 & 3 & 121 & oui & $110 \times 85 \times 30$ & 60 & SGD2 & $\begin{array}{l}\text { La matrice base silice est homogène et comprend de la fayalite en pavés et des } \\
\text { dendrites de wüstite éparses. Toutefois, les multiples différences de cristallisation } \\
\text { indiquent des cycles thermiques très irréguliers. Le métal est quasiment absent si } \\
\text { ce n'est localement dans le fond du culot sous forme de billes et filaments, } \\
\text { caractéristiques d'un métal en cours de brûlure. }\end{array}$ \\
\hline & & & & & & & & & $\begin{array}{l}\text { L'absence totale de pertes en métal indique sans doute le travail d'un objet bien } \\
\text { épuré, en fin d'élaboration. II faut remarquer que les battitures présentes dans ce } \\
\text { culot ne sont pas d'une taille aussi importante que dans les autres échantillons } \\
\text { étudiés. Cette indication tendrait à montrer que le travail ne s'effectuait pas sur de } \\
\text { petits objets. }\end{array}$ \\
\hline & & & & & & & & & $\begin{array}{l}\text { La matrice base silice, très homogène, contient des lattes de fayalite. Les pertes en } \\
\text { métal sont faibles et les battitures rares. L'oxydation à chaud est très faible. }\end{array}$ \\
\hline 000-000-001 & 0 & 0 & décapage & 242 & non & $95 \times 64 \times 53$ & ind & SGD & $\begin{array}{l}\text { Le travail de forge semble avoir été réalisé dans le cadre d'une bonne mâtrise de } \\
\text { l'oxydation à chaud. }\end{array}$ \\
\hline
\end{tabular}

Tableau 1 : Bilan des analyses métallographiques.

Table 1: Summary results of metallographic analyses. 
eux proviennent du groupe des SGD1 et le dernier est issu du groupe des SAS.

Dans l'ensemble des six culots étudiés provenant de cette phase, on observe une superposition de différentes formes de cristallisation de la fayalite et des rares pertes en oxydes sous la forme de globules ou dendrites de wüstite (fig. 2a). Cet état est le reflet d'un cyclage thermique caractéristique de multiples épisodes de chauffe traduisant une opération de forge.

Pour la phase la plus ancienne qui nous intéresse ici, les matrices base silice sont plutôt pauvres en wüstite (oxydes) (fig. 2b), à la différence des culots de la phase la plus récente dont les matrices en sont saturées (fig. 2c). À l'inverse, les culots les plus anciens ne contiennent que de rares dendrites et globules de wüstite épars révélateurs d'une faible oxydation à chaud. La quasi absence de pertes en oxydes, sous la forme de wüstite ou de battitures peut refléter un martelage peu intensif ou une bonne efficacité du processus. Les rares battitures rencontrées dans ces six culots sont de grandes dimensions (fig. $2 \mathrm{~d}$ ) indiquant alors une mise en œuvre de produits de tailles conséquentes. Le gabarit des battitures est en effet proportionnel à celui de l'objet travaillé. Ainsi, un objet de grande taille qui demande une chauffe plus longue ainsi qu'un choc mécanique plus important entraîne de fait l'apparition en surface du métal d'une couche d'oxyde plus conséquente que si le travail de forge concernait la réalisation de petits objets. Elles sont également majoritairement à tendances planes ce qui traduit plutôt un travail de produits plats (fig. 2e).

Quant au métal travaillé par les forgerons d'Orval, il est difficile d'en préciser les qualités car les pertes métalliques sont peu abondantes dans les culots. Seul l'un d'entre eux (131-24-29) a livré un fragment d'acier corrodé (fig. 3f), autour de $0,7 \%$ à $0,8 \%$ de carbone, dont la taille conséquente $(16 \times 6 \mathrm{~mm})$ laisse présager le travail d'un objet volumineux et corrèle les observations, sur la taille des matériaux travaillés, déjà notées à partir des battitures. La présence d'éclats de métal en cours de brûlure et de quelques billes (fig. 3g) sont d'autres indices du travail de l'acier. En effet, celui-ci brûle plus facilement que le fer (le point de fusion de l'acier, $1142{ }^{\circ} \mathrm{C}$ pour $2 \%$ de carbone à $1493^{\circ} \mathrm{C}$ pour $0,3 \%$ de carbone, est plus bas que celui du fer qui est à $1535^{\circ} \mathrm{C}$ ), ce qui conduit à la formation de ces billes caractéristiques.

Il semble que le travail de forge ait été bien maîtrisé car hormis quelques battitures, les pertes en oxydes (wüstite) sont très faibles et probablement révélatrices d'une maîtrise technique particulière des forgerons d'Orval. Ainsi la faible ampleur des épisodes de chauffes visait sans doute à ne pas brûler des objets aciérés de grandes tailles. De même, la présence rare de pertes métalliques évoque un travail de finition d'objets.

\section{OrVal dans le ConteXte Des habitats ENCLOS DU SECOND ÂGE DU FER}

Au sein des établissements ruraux enclos gaulois, les activités métallurgiques reconnues sur le site des Pleines paraissent-elles marginales ou au contraire doivent-elles être considérées comme de pratique courante? Pour répondre à cette question, nous avons dépouillé les Bilans Scientifiques Régionaux de Basse-Normandie. Seuls ont été intégrés dans cette étude les établissements enclos suffisamment documentés. Ainsi, les rares occupations " ouvertes " (Pont-Hébert, "le Rocher »; Marcei, « le Marais »...) n’ont pas été prises en compte. De même les sites uniquement connus par le biais de sondages ou par une approche trop partielle des structures ont eux aussi été écartés de notre corpus de base. À titre d'exemples, les établissements sondés sur le plateau de Thaon par Guy San Juan (San Juan et al., 1999) ou encore l'enclos repéré par Cyril Marcigny sur le site de Tatihou (Marcigny et Ghesquière, 2003) mais qui n’a été abordé que de façon très succincte à l'occasion de la fouille du parcellaire daté de l'âge du Bronze de l'île n'ont pas été retenus dans cette enquête.

Pour ce travail, 52 sites ont servi de base documentaire. Loin de présenter des caractéristiques extraordinaires, ces enclos semblent, en Basse-Normandie tout au moins, refléter l'unité domestique de base des campagnes gauloises (Jahier et Vauterin, 2011; Lepaumier et al., 2011b)

\section{La métallurgie du fer}

Dans notre corpus de référence, 35 sites ont livré des vestiges liés à la métallurgie du fer (fig. 3). Nous voyons ici l'importance de cette activité qui constitue, avec une présence sur $67 \%$ des sites, l'une des mieux représentées sur les enclos du second âge du Fer. Lorsque des études plus détaillées ont été réalisées, elles ont par ailleurs systématiquement identifié des culots de forge. Dans la région bas-normande, en l'état actuel, aucune activité de réduction (et donc de production) du fer n’a encore été identifiée.

Tout d'abord, nous constatons une variabilité assez importante des dimensions des scories. Si nous nous référons à la masse des culots entiers, les valeurs varient de 16 à $827 \mathrm{~g}$. Nous pouvons également remarquer que sur l'ensemble des corpus étudiés jusqu’à présent dans la région, seul un culot double de $706 \mathrm{~g}$, en plus de celui d'Orval, a été identifié sur le site de Falaise " Expansia " (Calvados, Besnard Vauterin, 2008).

Nous retrouvons bien évidemment ici les différentes grandes catégories de scories de forge et notamment les sco- 


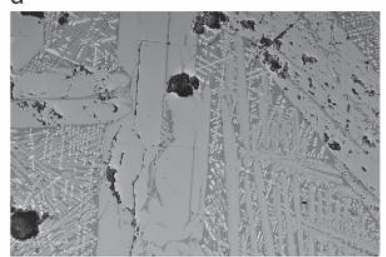

171-7-8

b

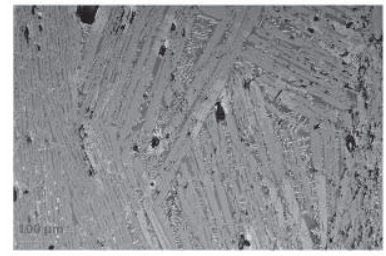

171-7-8

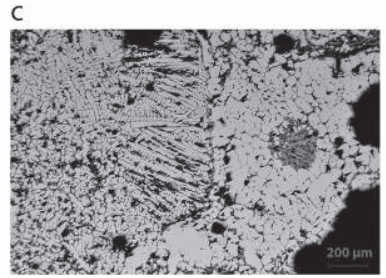

13-7-3

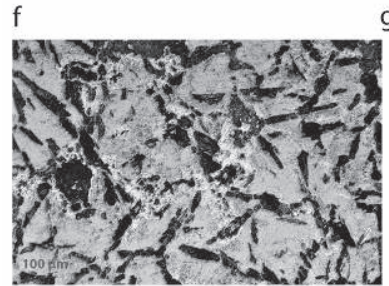

131-24-29

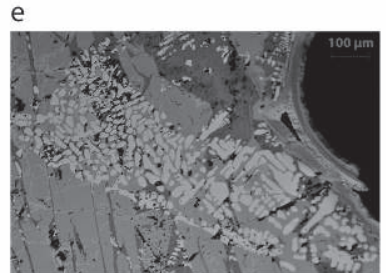

171-6-1

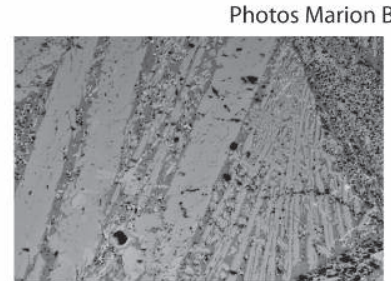

131-24-24

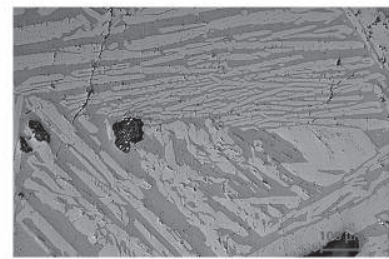

150-100-1

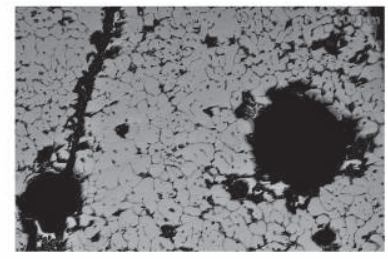

13-7-3

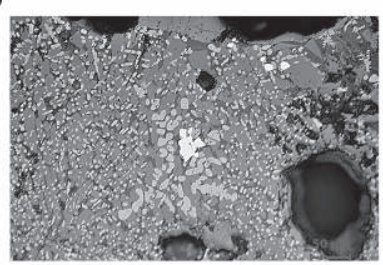

131-24-29

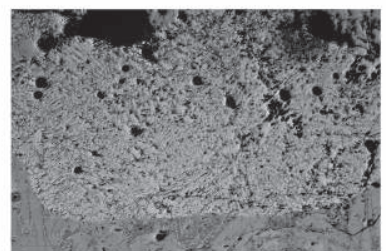

171-6-1

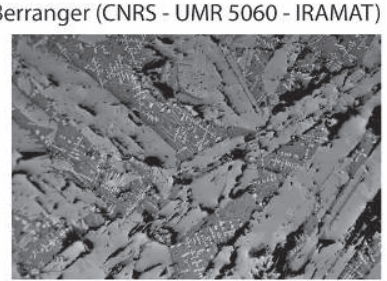

171-6-1

\section{d}

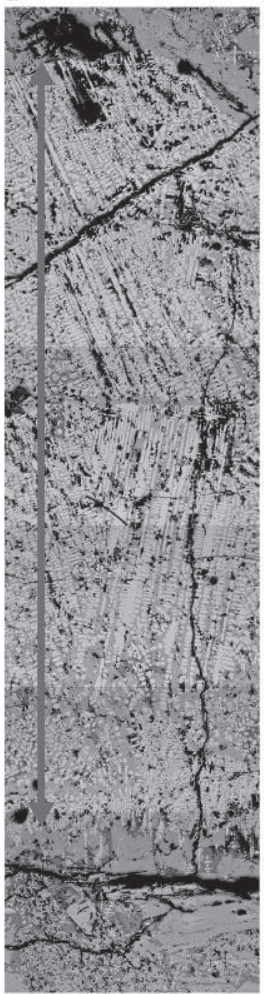

131-24-24

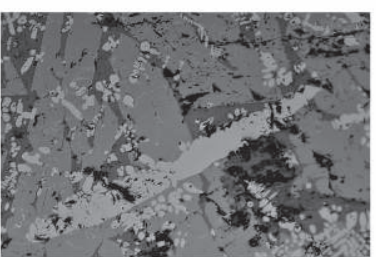

171-6-1
Figure 2 : (Voir planche couleur) a. Indices d'un cyclage thermique évoquant de multiples épisodes de chauffe et caractéristique d'une opération de forge; b. Matrices plutôt pauvres en wüstite (oxydes); c. Matrices saturées en wüstite (oxydes); d. Battitures de grandes tailles; e. Battitures majoritairement à tendance plane; $f$. Structures fantômes d'un fragment d'acier dans les produits de corrosion; g. Présence de fragments de métal en cours de brûlure et de quelques billes.

Figure 2: (See colour plate) a. Evidence of thermal cycling suggesting multiple stoking episodes characteristic of forging operation; b. Matrix with limited présence of wiustite; c. Wiistite saturated matrix; $d$. Large hammerscales; e. Mainly flat hammerscales; $f$. Ghost structures of a fragment of steel in corrosion material; g. Presence of metal beads and metal fragments in the burning process. ries argilo-sableuses qui sont légères, souvent informes et constituées pour une grande partie de matériaux argileux, les scories denses de couleurs grises et parfois rouillées qui se présentent généralement sous forme de calotte et les scories grises jaunâtre dense qui sont comme les précédentes avec en plus, en surface supérieure, un bourrelet vitreux. Ces différences de formes, de faciès, de densités, reflètent probablement la variabilité des activités. Ainsi, un site comme Marcei « le Maréchal » (Orne) (Jahier, 2005) livre essentiellement de grosses et lourdes scories en calottes alors que les sites de «l'Étoile » à Mondeville (Calvados) (Zaour, 2009b, p. 121122) ne semblent avoir produit que de petites scories. Parmi les hypothèses susceptibles d'expliquer ces disparités on peut évoquer la qualité des métaux travaillés mais aussi les masses de métal chauffées ou encore les temps et températures de forgeage (Fluzin et al., 2011, p. 230-231; Le Carlier et al., 2007, p. 29). Pour ce qui est des scories argilo-sableuses ou des scories grises jaunâtres denses, leur présence signe l'em- 


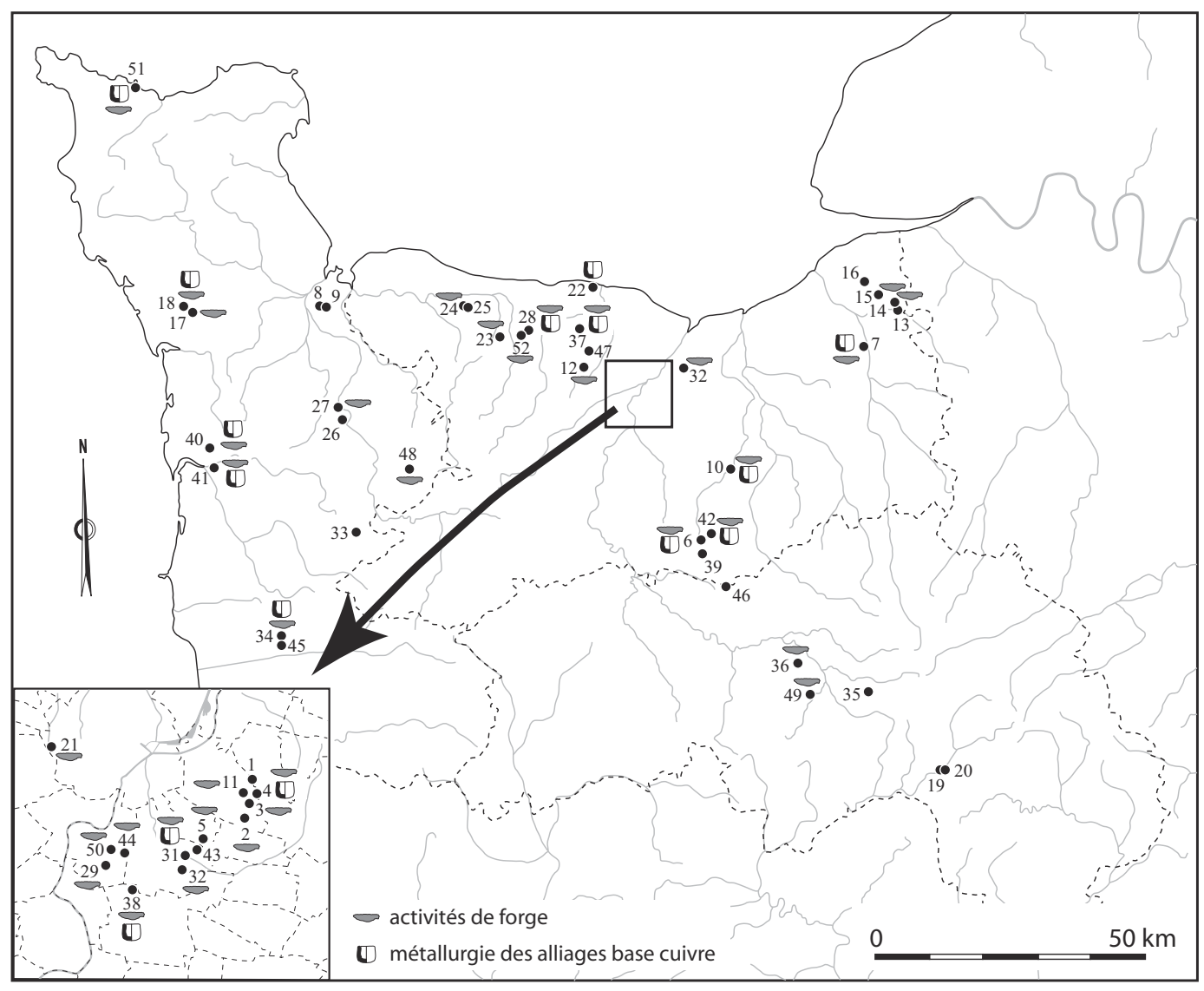

\begin{tabular}{rlcl}
$\mathrm{n}^{\circ}$ & commune & dépt & nom du site \\
\hline 1 & Mondeville/Giberville & 14 & le MIR \\
\hline 2 & Mondeville & 14 & le Haut-Saint-Martin \\
\hline 3 & Mondeville & 14 & L'Etoile 1 \\
\hline 4 & Mondeville & 14 & L'Etoile 2 \\
\hline 5 & Cormelles-le-Royal & 14 & Aire des Gens du Voyage \\
\hline 6 & Falaise & 14 & L'Attache \\
\hline 7 & Fierville-les-Parcs & 14 & Le Pré de la Val \\
\hline 8 & Saint Pellerin & 50 & La Fourchette 2 \\
\hline 9 & Les Veys & 50 & Le Haut Clos \\
\hline 10 & Condé sur Ifs & 14 & La Bruyère du Hamel \\
\hline 11 & Mondeville & 14 & L'Etoile 3 \\
\hline 12 & Putot-en-Bessin & 14 & Echangeur \\
\hline 13 & Quetteville & 14 & Les Heurtries II \\
\hline 14 & Quetteville & 14 & La Gohaigne \\
\hline 15 & Saint-Benoît d'Hébertot & 14 & Tontuit \\
\hline 16 & Saint Gatien-des-Bois & 14 & Le Vert Buisson \\
\hline 17 & Montgardon & 50 & La Bannerie \\
\hline 18 & Saint-Symphorien-le-Valois & 50 & La Valoiserie \\
\hline 19 & Buré & 61 & La Houssaye \\
\hline 20 & Buré & 61 & La Sarthe \\
\hline 21 & Caen & 14 & ZAC Beaulieu \\
\hline 22 & Courseulles-sur-Mer & 14 & La Fosse Touzé \\
\hline 23 & Barbeville & 14 & L'Entretenant \\
\hline 24 & Mosles & 14 & La Pièce du Pressoir \\
\hline 25 & Mosles & 14 & La Vignette \\
\hline 26 & Agneaux & 50 & Bellevue \\
\hline & & & \\
\hline & & \\
\hline
\end{tabular}

\begin{tabular}{llcl}
$\mathrm{n}^{\circ}$ & commune & dépt & nom du site \\
\hline 27 & Hébécrevon & 50 & La Couesnerie \\
\hline 28 & Saint-Martin-des-Entrées & 14 & Le Parc sur l'Herbage \\
\hline 29 & Fleury-sur-Orne & 14 & Parc d'Activités \\
\hline 30 & Hérouvillette & 14 & RD 513 \\
\hline 31 & Ifs & 14 & Object'Ifs Sud ens. 5 \\
\hline 32 & Ifs & 14 & Object'Ifs Sud ens. 6 \\
\hline 33 & Gouvets & 61 & La Bruyère de Rousseville \\
\hline 34 & Plomb & 50 & Le Champ du Puits \\
\hline 35 & Neuville-près-Sées & 61 & Les Ruisseaux \\
\hline 36 & Marcei & 61 & Le Maréchal \\
\hline 37 & Creully & 14 & Le Clos de l'Epinette \\
\hline 38 & Saint-Martin-de-Fontenay & 14 & La Grande Barberie \\
\hline 39 & Saint-Martin-de-Mieux & 14 & Vallembras \\
\hline 40 & Bricqueville-la-Blouette & 50 & La Roguerie \\
\hline 41 & Orval & 50 & Les Pleines \\
\hline 42 & Falaise & 14 & Expansia \\
\hline 43 & Ifs & 14 & AR 67 \\
\hline 44 & Fleury-sur-Orne & 14 & CD 120/ikéa \\
\hline 45 & Plomb & 50 & Le Pré en Pente \\
\hline 46 & Nécy & 14 & La Hoguette \\
\hline 47 & Thaon & 14 & site n ${ }^{\circ} 6$ \\
\hline 48 & Giéville & 50 & La Bigne \\
\hline 49 & Mortrée & 61 & Le Pré du Palluel \\
\hline 50 & Fleury-sur-Orne & 14 & Les mézerettes \\
\hline 51 & Nacqueville & 50 & La Batterie Basse \\
\hline 52 & Saint-Martin-des-Entrées & 14 & La Pièce des Côtelets \\
\hline & & & \\
\hline
\end{tabular}

Figure 3 : Carte de localisation des sites enclos du $2^{\text {nd }}$ âge du Fer fouillé en Basse-Normandie depuis 1991. Figure 3: Map of enclosed sites of the $2^{\text {nd }}$ Iron Age excavated in Lower-Normandy since 1991. 

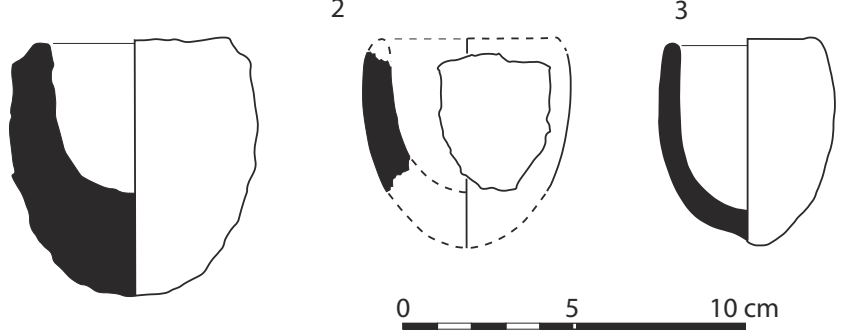

7

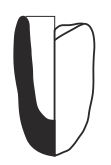

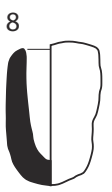

14

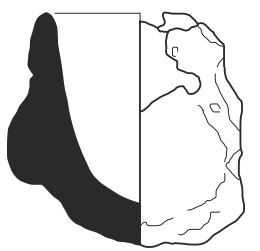

9

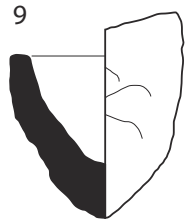

15

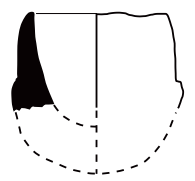

10

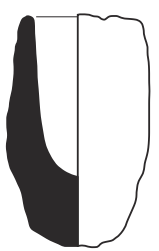

16
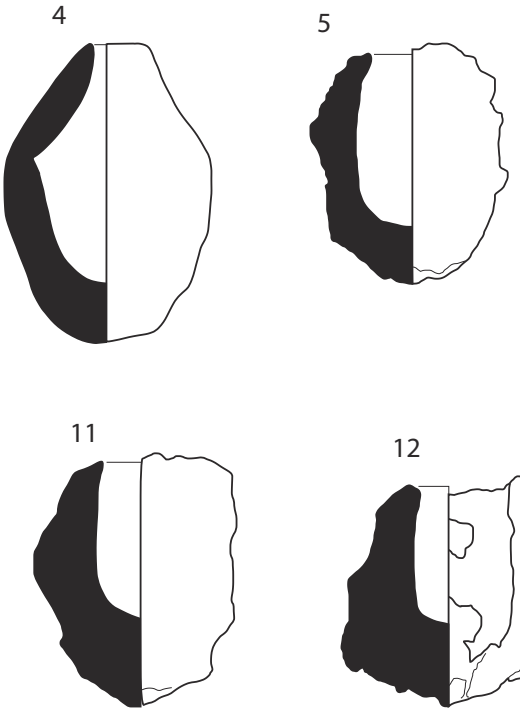

17

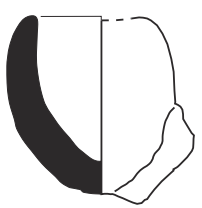

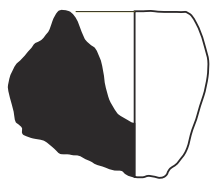
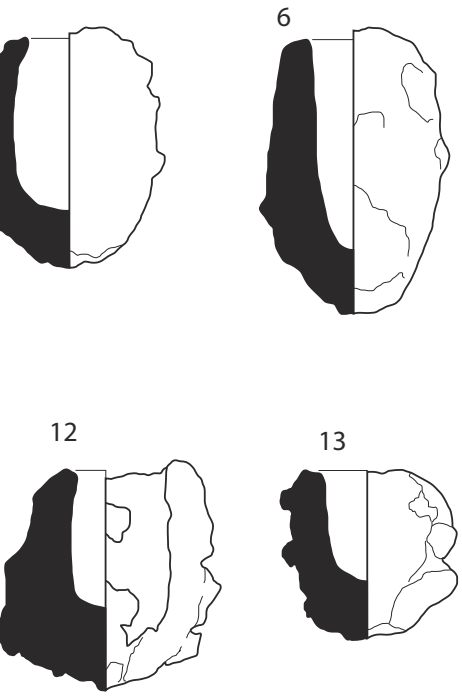

Figure 4 : Creusets découverts sur les sites enclos ruraux du $2^{\text {nd }}$ âge du Fer bas-normands. Saint-Symphorien-le-Valois, "La Valoiserie »; 2. Bricqueville, "La Blouette »; 3 et 4. Condé sur Ifs, "La Bruyère du Hamel »; 5 à 14 Ifs, "Object'Ifs Sud, ensemble 5 »; 15. Fontenai sur Orne, "La Patte d'Oie » 16. Orval, "Les Pleines »; 17. Mondeville, « l'Étoile »; 18 et 19. Falaise, " Expansia ».

Figure 4: Crucibles discovered at enclosed sites of the 2nd Iron Age in Normandy. 1. Saint-Symphorien-le-Valois, "La Valoiserie"; 2. Bricqueville, "La Blouette"; 3 et 4. Condé sur Ifs, "La Bruyère du Hamel"; 5 à 14 Ifs, "Object'Ifs Sud, ensemble 5"; 15. Fontenai sur Orne, "La Patte d'Oie" 16. Orval, "Les Pleines"; 17. Mondeville, "l'Étoile"; 18 et 19. Falaise, "Expansia".

ploi d'ajouts lors des activités de soudure (Le Carlier et al., 2007, p. 26-27) ou résulte de l'altération des parois du foyer suivant la qualité de l'argile utilisée (plus ou moins fusible) et la puissance de la ventilation.

La forme des culots est également sujette à d'importantes variations. Les culots sont le plus souvent circulaires, mais peuvent parfois se révéler quadrangulaires comme cela a pu être observé sur le site des "Heurtries II " à Quetteville (Calvados, Lepaumier, 1996) ou encore sur celui de Marcei «Le Maréchal » (Orne, Jahier, 2005). Ces différences apportent également des informations sur la forme des foyers qui les ont engendrés.

Les scories ne sont pas les seuls éléments qui peuvent traduire la présence d'une activité de travail du fer sur ces établissements. La présence de demi-produits ou d'outils spécifiques peut également y contribuer. Ainsi une pince de forgeron a été découverte sur le site de Giéville " $\mathrm{La}$ Gohaigne "(Manche, Vauterin, 2011, p. 214-215) alors même qu'aucune scorie n'a été identifiée sur la partie de l'enclos qui a pu être explorée. Quelques tas sont identifiés comme par exemple sur le site de Nacqueville dans la Manche (Lefort et Marcigny, 2009), ou encore sur celui de Fleury-sur-Orne «ZL 7 » dans le Calvados (Lepaumier et al., à paraître). Enfin, plusieurs demi-produits ont été reconnus sur ces établissements. Si pour l'instant seul le currency-bar de Cormelles-le-Royal (Calvados, Carpentier et al., 2002) a pu bénéficier d'une étude métallographique révélant son obtention par soudure de deux masses métalliques incomplètement épurées (15 à $20 \%$ d'inclusions subsistant dans la matrice métallique) et majoritairement aciérées autour de 0,2 \% C (Berranger 2013, p. 225-226), au moins 3 autres barres à douille semblent pouvoir prétendre à l'appellation de currency-bars. Ces objets ont été identifiés au sein des collections des sites du "MIR »(Calvados, 2 exemplaires) et d'Ifs "Object'Ifs sud ens. 6 "(Calvados). Ces données peuvent être rapprochées de la découverte d'un dépôt de 70 demi-produits de ce type sur la commune voisine de Bretteville-sur-Odon (Calvados, Feugère, 2000). Une autre 
barre est signalée comme un possible currency-bar sur le site de Nacqueville (Manche, Lefort et Marcigny, 2009). Un autre objet, reconnu sur le site d'Ifs " Object'Ifs sud ens 5 " (Calvados), pourrait s'apparenter à un demi produit différent, de type hooked billet. Par rapport aux exemplaires connus qui proposent des masses comprises entre 1 et $3 \mathrm{~kg}$ (Crew, 1994; Berranger et Fluzin, 2011), il convient toutefois de noter ici que les dimensions de l'objet sont plus faibles. Enfin, en marge de l'habitat de Nacqueville (Manche) un demi-produit de type bipyramidé aujourd'hui disparu a été signalé par Georges Rouxel au début du xxe siècle (Lefort et Marcigny, 2009, p. 71).

\section{La métallurgie des alliages cuivreux}

Cette activité métallurgique est moins bien représentée que celle du fer mais apparaît néanmoins sur 15 sites. Elle est le plus souvent perçue par la présence de creusets, plus rarement par celle de moules (Saint-Martin-des-Entrées, Marcigny et al., 2004), de déchets de coulée (Fleury-surOrne, "ZL 7 », Lepaumier, 2012) ou encore de scories spécifiques (Fierville-les-Parcs, Jahier et al., 2002). Le nombre de creusets est variable mais se limite le plus souvent à quelques unités. De rares sites comme celui d'Ifs "Object'Ifs sud ens. 5 " (Calvados) dérogent à cette règle. Parmi les 12 creusets du site dont 10 étaient regroupés dans le comblement d'un fossé (Le Goff, 2002), deux se démarquent nettement. De très petites dimensions, ils présentent un volume de seulement $4 \mathrm{~cm}^{3}$ et une paroi externe sans vitrification, ce qui pourrait les apparenter davantage à des creusets d'orfèvres plutôt qu’à ceux de bronziers. Le site de Condé-sur-Ifs, « la Bruyère du Hamel » (Calvados) a quant à lui livré 7 creusets. Parmi ceux-ci, deux types ont clairement pu être distingués (Dron et al., 2011). Le premier qui compte 3 représentants correspond à un type récurrent pour l'âge du Fer, de forme ovoïde à ouverture large. Le second, reconnu à 4 exemplaires, est de taille un peu plus importante mais surtout propose un profil très particulier, avec une ouverture très rétrécie qui lui confère l'aspect d'une goutte d'eau ou d'une grenade.

On ne sait pas exactement quels objets ont pu être produits sur ces sites, mais les creusets présentent d'assez faibles dimensions (fig. 4). D'un volume utile compris entre 4 et $63 \mathrm{~cm}^{3}$, ils ne pouvaient permettre que la production de petits objets. Les objets en bronze rencontrés sur ces habitats sont d'ailleurs assez peu nombreux et de petites dimensions. Il s'agit pour l'essentiel de monnaies, de parures (fibules et bracelets) et de divers petits mobiliers (pince à épiler, rivets...). On peut toutefois noter qu'en dehors du site de Courseulles-sur-Mer, "La Fosse Touzé », établissement quelque peu particulier au sein de notre corpus (Jahier, 2011), le travail des alliages cuivreux cohabite systématiquement avec celui des ferreux.

\section{Les foyers}

Enfin, les activités métallurgiques se traduisent quelquefois sur les sites par la présence de parois de foyers scoriacées. Le seul foyer en place clairement identifié dans la région a été étudié sur le site de Marcei dans l'Orne (Jahier, 2005; Zaour, 2009a). Localisé à $1 \mathrm{~m}$ de l'emplacement présumé de l'enclume, il se présentait sous une forme quasi-circulaire de 22 à $24 \mathrm{~cm}$ de diamètre pour une profondeur conservée sous le décapage de $18 \mathrm{~cm}$. En dehors de cette structure de combustion, 5 sites (Quetteville et Falaise dans le Calvados, Marcei dans l'Orne et Bricqueville-la-Blouette et Orval dans la Manche) ont livré des fragments de parois portant encore l'empreinte d'un ou plusieurs trous à vent. Sur les 10 éléments suffisamment bien préservés pour permettre une prise de mesure, leurs diamètres varient de 1,2 à 2,4 cm (fig. 5a). Trois de ces éléments présentent non pas une empreinte unique mais deux perforations. À Marcei, le fragment possède une base plane et deux empreintes cylindriques d'environ $2 \mathrm{~cm}$ de diamètre. Ces empreintes parallèles occupent une position légèrement oblique par rapport à la base et débouchent sur une surface scoriacée. À Orval, un fragment de paroi scoriacée porte la trace d'un double trou à vent. Les évents sont cassées et le diamètre le plus important qui est encore visible est de $1,5 \mathrm{~cm}$. Des coulées de scories sont visibles sous les deux évents sur la paroi interne. Les canaux des évents sont vitrifiés et de couleur rose à noire en passant par du vert sur la face interne. Celle-ci est scoriacée de couleur jaune et la face opposée qui n'est pas vitrifiée est de couleur rouge à violette. À Bricqueville « la Blouette", deux éléments s'appareillent et se recollent pour ne former qu'un seul fragment en bordure duquel deux empreintes de trous à vents se dessinent. L'ensemble de ces éléments, offrant deux empreintes mitoyennes, illustre l'utilisation d'un double soufflet permettant d'assurer une ventilation continue au sein des foyers de forge.

Deux éléments semblent correspondre à des tuyères. Découverts sur les sites d'Ifs, "Object'Ifs sud, ensemble 5 " (où le fragment est associé aux 10 creusets du fossé 793, Le Goff, 2002) et d'Orval «Les Pleines ", ils présentent tous deux la même physionomie. Il s'agit de tubes d'argiles de quelques centimètres de longueur ménageant, dans le cas d'Orval, un conduit interne de 1,7 cm (fig. 5b). 
a

\begin{tabular}{lccccc} 
Site & Poids (en g) & Long. (en cm) & Larg. (en cm) & Ep. (en cm) & Diam estimé (en cm) \\
\hline Falaise, "Expansia" & 19 & 4,9 & 4 & 1 & 2,3 \\
\hline Bricqueville, "La Blouette" & 97 & 10 & 5 & 2,8 & $2,5(\mathrm{x} 2)$ \\
\hline Orval, "Les Pleines" & 48 & 8,3 & 4,9 & 1,6 & $1,5(\mathrm{x} 2)$ \\
\hline Orval, "Les Pleines" & 38 & 6,3 & 5,1 & 1,4 & 1,2 \\
\hline Orval, "Les Pleines" & 23 & 5 & 3,7 & 1,3 & 1,6 \\
\hline Orval, "Les Pleines" & 10 & 2,5 & 2,6 & 1,8 & 1,3 \\
\hline Orval, "Les Pleines" & 26 & 5,6 & 3,3 & 1,4 & 2,2 \\
\hline Orval, "Les Pleines" & 44 & 5,3 & 4,1 & 2 & 2,2 \\
\hline Marcei "Le Maréchal" & 88 & 6,9 & 5,2 & 4,7 & $2,0(x 2)$ \\
\hline Quetteville, "Les Heurtries II" & 85 & 9,4 & 7,2 & 2,4 & 2,4
\end{tabular}

b
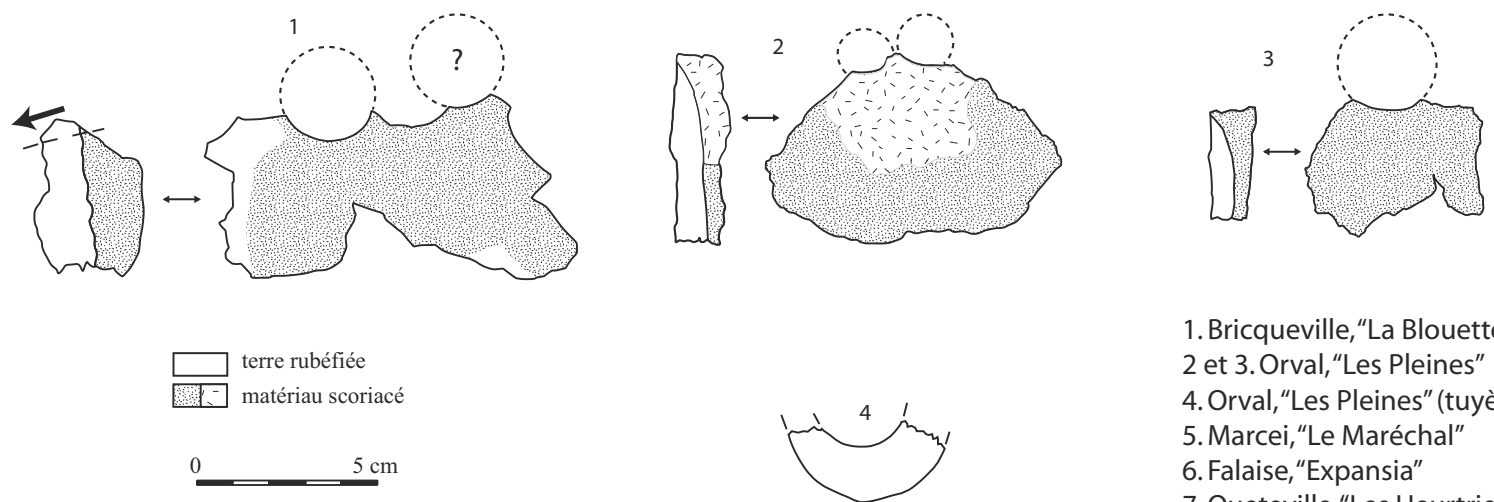

1. Bricqueville,"La Blouette" 2 et 3. Orval, "Les Pleines" 4. Orval, "Les Pleines" (tuyère) 5. Marcei,"Le Maréchal”

6. Falaise, "Expansia"

7. Queteville,"Les Heurtries II"
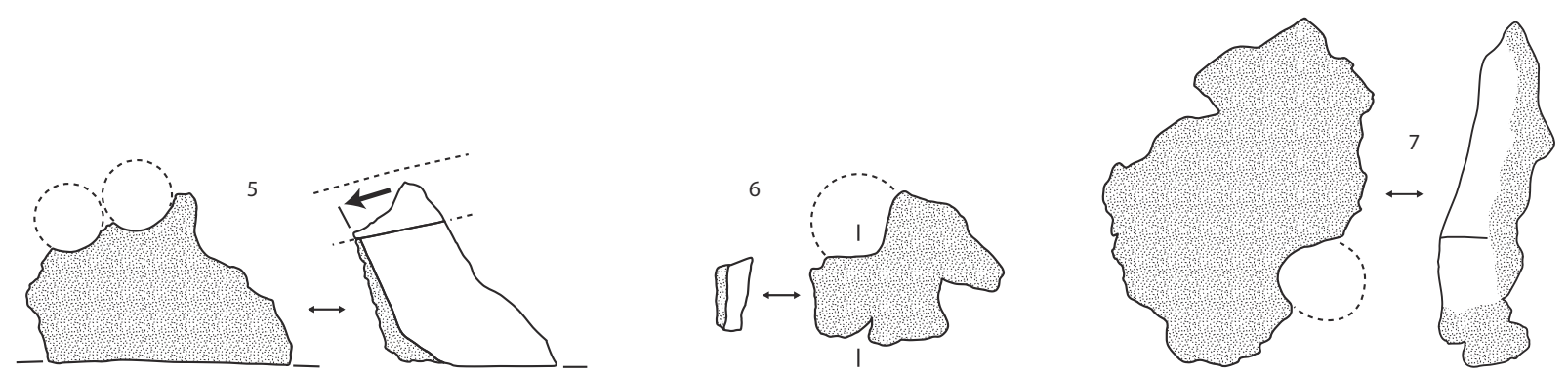

Figure 5 : a. Relevé des dimensions des éléments de ventilation d'Orval, Briqueville, Falaise, Marcei, Quetteville; b. Exemples d'éléments de ventilation d'Orval, Bricqueville, Falaise, Marcei, Quetteville.

Figure 5: a. Dimensions of the ventilation ducts from Orval, Briqueville, Falaise, Marcei, Quetteville; b. Examples of ventilation ducts from Orval, Bricqueville, Falaise, Marcei, Quetteville.

\section{BILAN}

Au terme de cette étude plusieurs constats s'imposent. Tout d'abord, aucun vestige ne permet pour l'instant d'envisager la production du fer (réduction) sur les sites domes- tiques enclos bas-normands. Ce constat rejoint celui établit plus largement lors de l'enquête nationale conduite sous la direction de François Malrain, Geertrui Blancquaert et Thierry Lorho à l'occasion du colloque AFEAF de Chauvigny (Malrain et al., 2009, p. 37). 
Ainsi, seuls des déchets liés aux activités de post-réduction y sont retrouvés. Cependant, en l'absence d'analyses métallographiques systématiques réalisées sur l'ensemble du corpus, il n'est, à ce jour, pas possible de mieux caractériser cette activité et de distinguer des différences dans les pratiques de forgeage. L'aboutissement d'une thèse sur la métallurgie du fer en Normandie et ses marges de l'âge du Fer au Moyen Âge (Zaour en cours) permettra sans doute de lever le voile sur certaines des questions qui se posent quant à la qualité des métaux travaillés sur ces sites, la dextérité et la qualification des forgerons qui ont utilisé ces ateliers.

Ce qu'il est possible, d'ores et déjà, d'affirmer c'est que les déchets sidérurgiques sont très largement répandus sur les sites du corpus. En termes d'occurrence, ils illustrent sans doute l'une des activités artisanales les mieux représentées dans les campagnes gauloises. Sans devoir nécessairement être généralisée à l'ensemble des régions septentrionales de la Gaule, on retrouve ici une observation déjà réalisée ailleurs. Par exemple dans le sud-est du Bassin parisien (Dunikowski et al., 2007) ou encore dans la région des sources de la Seine, en Côte-d'Or, où un tiers des établissements ruraux attribués à la fin de La Tène finale ou au début du haut-Empire ont livré des déchets de forge (Mangin et Fluzin, 2006). Si l'on se réfere au schéma envisagé pour la Picardie (Beauvais et al., 2007), un site comme celui d'Orval pourrait parfaitement s'intégrer comme site de niveau 3 qui concerne les établissements où le travail du métal se cantonne à une production réduite d'objets simples. Du point de vue quantitatif, les volumes restent d'ailleurs assez modestes et sont sans commune mesure avec ceux enregistrés sur les habitats groupés contemporains. Un simple dépouillement bibliographique permet de mieux cerner cette dualité (tab. 2). Sur les habitats ruraux de type «ferme indigène » ou apparenté, les quantités se limitent à quelques kilogrammes de scories alors qu'elles atteignent plusieurs dizaines voire plusieurs centaines de kilogrammes sur les habitats groupés contemporains. Par exemple, à Bobigny Sylvain Bauvais a décompté (Bauvais, 2007, p. 184-203) plus de $60 \mathrm{~kg}$ de déchets sidérurgiques. À Varennes sur Seine, site qui n'a pas été fouillé en intégralité, ce sont plus de $512 \mathrm{~kg}$ de scories qui ont été reconnus (Dunikowski et al., 2007). Ces différences traduisent la distinction que J.-P. Guillaumet soulignait entre artisan de proximité et artisan de production (Guillaumet, 1996). Cette abondance de forge, y compris dans des réseaux de fermes aussi denses que celui de la périphérie caennaise où les sites contemporains ne sont distants de quelques centaines de mètres, permet même d'évoquer l'activité de " paysans forgerons ». Sur la plupart de ces sites il faut sans doute imaginer des forges occasionnelles nécessaires au bon fonctionnement du site et susceptibles de produire les objets les plus simples. Les pièces manufacturées ont dû être peu nombreuses et leur fabrication ne devaient pas requérir une dextérité particulière de la part de ces "paysans forgerons " (clous, crampons d'assemblage, couteaux, outillage de base...). Quoi qu'il en soit, la diversité de taille et de forme des culots semble marquer une certaine polyvalence des travaux exécutés. Si l'on s'inscrit dans le système établi par Vincent Serneels pour l'époque antique il faut voir sur ces sites des forges de service (Serneels, 1998), ce que Christophe Pellecuer (Pellecuer, 1998, p. 173) a pu dénommer comme "ateliers de circonstances".

Au même titre que la plupart des agriculteurs actuels mầtrisent les techniques de base du soudeur, il est probable que les paysans gaulois devaient connaître les techniques de base du forgeron. Il faut d'ailleurs noter à ce sujet qu'il est assez simple de forger un clou ou une agrafe, voire même de produire une lame à simple tranchant (couteau, faucille...) à partir d'un demi-produit de type currency bar. La notion de forge d'entretien (Guillaumet et Nillesse, 2000) souvent mise en avant pour les activités métallurgiques associées à ces établissements enclos suppose quant à elle des gestes plus techniques comme la maîtrise de la soudure pour réparer un outil ou toute autre pièce métallique cassée ou déchirée. En revanche, il semble assez évident, au vu des recherches actuelles, que les artisans véritablement spécialisés n’officiaient pas dans ces établissements ruraux mais devaient produire au sein d'ateliers localisés sur les habitats groupés.

Le plus surprenant en définitive au terme de cette étude concerne la présence assez répandue de déchets du travail des métaux fusibles. En effet, le travail des métaux fusibles comme les alliages cuivreux demande de nombreux savoirfaire pour arriver à l'objet fini. Ainsi, outre la maîtrise du foyer (qui n'est guère différente de celle nécessaire à la forge avec des températures similaires dans les deux cas : environ $1100^{\circ} \mathrm{C}$ ), il faut aussi contrôler toutes les étapes depuis la fabrication du moule, la cuisson et le décirage de celui-ci, la préparation de l'alliage, la coulée et le polissage de l'objet fini. Par rapport aux activités de forge, ces techniques sont par ailleurs utilisées non plus dans un but purement fonctionnel, mais bien davantage dans un dessein souvent plus accessoire. Si les fibules, anneaux ou autres rivets permettent d'affirmer un certain statut social, les objets en alliages base cuivre n'avaient pas la même utilité que les objets en fer plutôt employés dans les domaines domestique et agricole. Pour ces travaux délicats d'orfevrerie, à l'instar de ce que certains envisagent (Ménez, 1996, p. 143), l'hypothèse d'un artisan itinérant se déplaçant de ferme en ferme ne peut donc être totalement écartée.

Enfin, la large diffusion de ces techniques métallurgiques sur les sites d'habitats suppose un réseau d'approvisionne- 


\begin{tabular}{|c|c|c|c|c|}
\hline localité & département & nom du site & datation & quantité de scories (gr) \\
\hline Fleury-sur-Orne & 14 & Les Mézrettes & LTM/LTF & 28 \\
\hline Mondeville & 14 & L'étoile site 1 & LTM/LTF & 61 \\
\hline Bricqueville la Blouette & 50 & La Roguerie & LTA/LTM & 193 \\
\hline Mosles & 14 & La pièce du Pressoir & LTA & 352 \\
\hline Cormelles le Royal & 14 & Aire des Gens du Voyage & LTM/LTF & 527 \\
\hline Fierville-les-Parcs & 14 & Le Pré de la Val & LTA & 710 \\
\hline Hébécrevon & 50 & La Couesnerie & haut-Empire & 715 \\
\hline Écouflant & 49 & La Reculière & LTM & 800 \\
\hline St Pellerin / Les Veys & 50 & RN 174 / RN 13 & LTF & 844 \\
\hline Fleury-sur-Orne & 14 & ZL 7 & LTM/LTF & 849 \\
\hline Barbeville & 14 & L'Entretenant & LTM/LTF & 957 \\
\hline Bazoches-sur-Vesles & 2 & Les Chanteraines & LTM/LTF & 1153 \\
\hline Marcé & 49 & Le Deffroux & LTM/LTF & 1362 \\
\hline Bazoches les Bray & 77 & La Voie Neuve & LTF & 1400 \\
\hline Mondeville & 14 & L'étoile site 2 & LTM/LTF & 1403 \\
\hline Mondeville & 14 & L'étoile site 3 & LTM/LTF & 1822 \\
\hline Marcé & 49 & Hélouine & LTM/LTF & 2519 \\
\hline Falaise & 14 & ZAC Expansia, phase 2 & LTM/LTF & 2830 \\
\hline Quetteville & 14 & Les Heurtries II & LTM/LTF & 2850 \\
\hline Fontenay-le-Comte & 85 & Les Genâts & LTM/LTF & 2960 \\
\hline Meung-sur-loire & 45 & Le Clos Fleury & LTM/LTF & 3000 \\
\hline Fleury-sur-Orne & 14 & Parc d'Activités & LTM/LTF & 3105 \\
\hline Marcei & 61 & Le Maréchal & LTM/LTF & 3845 \\
\hline Bazoches les Bray & 77 & Près le Tureau aux Chèvres & LTM & 4000 \\
\hline Guichainville & 27 & Le Long Buisson & LTM/ausgustéen & 4143 \\
\hline Creully & 14 & Le Clos de l'Epinette & LTM/LTF & 4200 \\
\hline Laniscat & 22 & Le Haut Querrault & toutes phases confondues & 6250 \\
\hline Croixrault & 80 & L'aérodrome & LTM/LTF & 6259 \\
\hline Notre dame de l'Isle & 27 & La Plaine du Moulin à Vent & $\mathrm{LTM} / \mathrm{LTF}$ & 7786 \\
\hline Orval & 50 & Les Pleines & LTA/haut-Empire & 8509 \\
\hline Lamballe & 22 & La Tourelle site 1 & LTF & 10940 \\
\hline Rue & 80 & le Chemin des Morts & LTM/LTF & 12000 \\
\hline Ronchères & 2 & le bois de la Forge & LTM/LTF & 12718 \\
\hline Evreux & 27 & Cambolle / Le Golf & LTF & 14730 \\
\hline Vivoin & 72 & La Fosse & LTM/LTF & 16300 \\
\hline Dissay-sous-Courcillon & 72 & Beauregard & LTF & 18000 \\
\hline Varennes sur Seine & 77 & les Marais du Pont & LTM/LTF & $>512000$ \\
\hline Bobigny & 92 & La Vache à l'Aise / Avicennes & LTM/LTF & 61700 \\
\hline Levroux & 36 & Les Arènes & LTM/LTF & $>1500000$ \\
\hline
\end{tabular}

Tableau 2 : Tableau illustrant les quantités de scories découvertes sur un certain nombre de sites enclos du $2^{\text {nd }}$ âge du Fer du nord de la France. 1 re partie du tableau : sites enclos ruraux. $2^{\text {nd }}$ partie du tableau : habitat groupé. En gras : sites bas-normands.

Table 2: Table showing the quantities of slag discovered on a number of enclosed sites from the $2^{\text {nd }}$ Iron Age of Northern France. 1st part of the table: rural enclosed settlements. 2nd part the table: grouped settlement. Bold: Normandy sites. 
ment en combustible et en métal. En effet si les températures de plus de $1000{ }^{\circ} \mathrm{C}$ semblent pouvoir être atteintes avec du bois, l'utilisation de charbon de bois est grandement préconisée pour atteindre cette gamme de chaleur dans des foyers qui semblent-ils demeurent réduits à tout au plus quelques décimètres de diamètre. De plus, même si une partie du métal a pu être récupérée sur place (grappage, Dunikowski et al., 1996, p. 120, refonte des métaux précieux), il semble évident qu'une part importante des métaux a été importée sur les sites comme l'illustre la présence de plusieurs demiproduits. Or pour l'instant aucun site de réduction du fer n'a été reconnu dans la région. Si la forme des demi-produits les rattache majoritairement à la famille des currency-bars, un autre s'apparente à un hooked billet (Berranger et Fluzin, 2011). Ces deux formes, d'abord décrites outre Manche (Crew, 1994) apparaissent de plus en plus fréquentes sur la façade atlantique et le nord de la Gaule (Berranger et Fluzin, 2011; Berranger, 2013). Il est de ce fait difficile de préciser l'origine de ces demi-produits : Grande Bretagne ou continentale? D'une part, depuis les travaux de Barry Cunliffe, les relations avec le sud de l'Angleterre ne sont plus à démontrer (Cunliffe et De Jersey, 1997), d'un autre côté la Basse-Normandie est environnée de régions productrices de fer. À l'ouest des sites de production sont signalés dans le bassin qui s'étend depuis l'estuaire de la Rance à la forêt de Paimpont, au sud sur le secteur du Mans qui est en passe de devenir l'un des principaux secteurs sidérurgiques du nord de la Gaule pour les périodes gauloises et gallo-romaines; vers l'est avec de petits pôles de production dans les régions d'Évreux et du Pays de Bray où elles ont fait l'objet de nombreux travaux (Crew, 1994). Enfin, une importation plus lointaine depuis, par exemple, le secteur des Clérimois et du Pays d'Othe ne peut être écartée. On perçoit donc bien que les activités métallurgiques de ces paysans forgerons se sont nécessairement inscrites dans des circuits d'échanges plus vastes. Les études systématiques des principaux demiproduits en particulier par analyse des inclusions contenues dans le métal (en cours au LMC - UMR 5060 - CNRS), devraient apporter à ce sujet, prochainement, de nombreuses précisions régionales.

\section{Bibliographie}

BauvaIs S., 2007. Évolution de l'organisation des activités de forge dans le nord du Bassin parisien au second Âge du fer. Étude pluridsciplinaires de la chaîne opératoire en métallurgie du fer. Thèse de doctorat, UTBM, université de Franche-Comté, École Doctorale "Langages, espaces, temps, sociétés ». 2 volumes, 604 p. et 490 p.
Bauvais S., Gaudefroy S., Gransar F., Malrain F., Fluzin P., 2007 : Premières réflexions sur l'organisation des activités de forge en contexte rural à La Tène finale en Picardie. In P.-Y. Milcent (dir.). L'économie du fer protohistorique : de la production à la consommation du métal. Actes du XXVIII e colloque international de l'Association Française pour l'Étude de l'âge du Fer (Toulouse, mai 2004), Aquitania, supplément 14/2, 239-261.

Berranger M., 2013. La circulation des matières premières métalliques (métal brut, demi-produits) aux âges du Fer : modalités d'organisation des productions et de transmission des savoirfaire. In A. Colin et F. Verdin (dir.). L'âge du Fer en Aquitaine et sur ses marges. Mobilité des hommes, diffusion des idées, circulation des biens dans l'espace européen à l'àge du Fer. Actes du XXXVe colloque international de l'Association Française pour l'Étude de l'âge du Fer (Bordeaux, juin 2011), Aquitania, supplément $30,380 \mathrm{p}$.

Berranger M., Fluzin P., 2011. Du métal brut au demi-produit. Propriétés et modes de circulation des matières premières ferreuses. Aspects de la romanisation dans l'Est de la Gaule. Bibracte, $21: 877-886$.

Besnard Vauterin C.-C. (dir.), 2008. Falaise "Expansia " (Calvados). Un habitat du second âge du Fer et des vestiges de l'Antiquité et du Haut Moyen Âge. Rapport Final d'Opération, INRAP, SRA DRAC de Basse-Normandie, 85 p + annexes.

Carpentier V., Marcigny C., Savary X., Ghesquière E., 2002. Enclos et souterrain du second âge du fer dans la plaine de Caen, l'exemple de Cormelles le Royal (Calvados), Revue Archéologique de l'Ouest, 19 : 37-60.

Crew P., 1994. Currency Bars in Great-Britain. Typology and function. Les lingots en fer protohistoriques en Grande Bretagne, typologie et fonction. In M. Mangin (dir.). La sidérurgie ancienne de l'est de la France dans son contexte européen. Archéologie et archéométrie. Actes du colloque de Besançon (1013 nov 1993). Annales littéraires de l'Université de Besançon, 536, série archéologie, 40, 345-350.

Cunliffe B., De Jersey P., 1997. Armorica and Britain, CrossChannel relationships in the late first millennium BC. Studies in Celtic Coinage, Number 3. Oxford University Committee for Archaelogy Monographs, 45, 117 p.

Dunikowski C., Séguier J.-M., Caвboї S., 2007. La production du fer protohistorique au sud-est du bassin Parisien. In P.-Y. Milcent (dir.). L'économie du fer protohistorique : de la production à la consommation du métal. Actes du XXVIII ${ }^{e}$ colloque international de l'Association Française pour l'Étude de l'âge du Fer (Toulouse, mai 2004), Aquitania, supplément 14/2, 279-289.

Dunikowski Ch., Leroy M., Merluzzo P., Ploquin A., 1996. L'atelier de forge gallo romain de Nailly les Bordes (Yonne), 
contribution à la caractérisation des déchets de production. Revue Archéologique de l'Est, 47 : 97-121.

Dron J.-L., Baudry A., Clément-Sauleau S., Gâche D., Marcigny C., Zaour N., 2011. La Bruyère du Hamel à Condé-sur-Ifs : un site de La Tène ancienne dans la Plaine de Caen. In P. Barral, B. Dedet, F. Delrieu, P. Giraud, I. Le Goff, S. Mario, A. Villard-Le Tiec. L'âge du Fer en Basse-Normandie. Gestes funéraires en Gaule au second âge du Fer. Actes du XXXIII colloque international de l'Association Française pour l'Étude de l'âge du Fer (Caen 2009). Annales Littéraires de l'Université de Franche-Comté, Presses Universitaires de Franche-Comté, 883 , série « environnement, société et archéologie ", 14, vol. 1, 159-166.

Feugère M., 2000. Un nouveau dépôt de lingot de fer de La Tène finale : Bretteville sur Odon (Calvados), Instrumentum, $11: 15$.

Fluzin P., Beauvais S., Berranger M., Pages G., Dillmann P., 2011. The multidisciplinary approach (archaelogy and archaeometry) to bloomsmithing activities in France : example results from the last twenty years. In J. Hosek, C. Henry, L. Mihok (ed.). The archaeometallurgy of iron. Recent developments in archaeology and scientific research. Dedicated to Professor Radomir Pleiner. The Institute of Archaeology of the ASCR, Prague, 223-236.

Fluzin P., Ploquin A., Dabosi F., 2004. Approches métallurgiques et archéométriques. In M. Mangin (dir.). Le fer Éditions Errance (archéologiques), Paris, 113-174.

Guillaumet J.-P., 1996. L'artisanat chez les Gaulois. Éditions Errance, collection des Hespérides, 127 p.

Guillaumet J.-P., Nillesse O., 2000. Les petits objets de quelques fermes gauloises : approche méthodologique. In $\mathrm{S}$. Marion et G. Blancquaert (éd.) Les installations agricoles de l'âge du Fer en France septentrionale. Éditions ENDS rue d'Ulm. Collection Études d'Histoire et d'Archéologie, 6, 251-276.

JAhier I. (dir.), 2011. L'enceinte des premier et second âge du Fer de La Fosse Touzé (Courseulles-sur-Mer, Calvados). Entre résidence aristocratique et place de collecte monummentale. Document d'Archéologie Française, 104 (Paris MSH), 243 p.

JAhier I. (dir.), 2005. A88 Marcei/Boissy-La-Lande "Le Maréchal » (Orne), Rapport Final d'Opération, INRAP, SRA DRAC de Basse-Normandie, 77 p + annexes.

JAhier I., VAUTERIN C.-C. (avec la collaboration de M. Besnard), 2011. Formes et composantes de l'habitat à l'âge du Fer en Basse-Normandie : architecture, chronologie, organisation, statut - un premier bilan. In P. Barral, B. Dedet, F. Delrieu, P. Giraud, I. Le Goff, S. Mario, A. Villard-Le Tiec. L'âge du Fer en Basse-Normandie. Gestes funéraires en Gaule au second âge du Fer. Actes du XXXIII ${ }^{e}$ colloque international de l'Association Française pour l'Étude de l'âge du Fer (Caen 2009). Annales Littéraires de l'Université de Franche-Comté, Presses
Universitaires de Franche-Comté, 883, série « environnement, société et archéologie ", 14, vol 1, 95-137.

Jahier I., Méniel P., Ozouf J.-C., 2002. Une petite ferme de La Tène ancienne au "Pré de la Val » à Fierville-les-Parcs (Calvados). Revue Archéologique de l'Ouest, 19 : 15-36.

Leblanc J.-C., 2001. archéométrie des battitures de forge. In L'obtenció del ferro pel procediment directe entre els segles IV i XIX, actes du 6e colloque d'archéologie d'Andorre, 2000, Ministeri de Cultura, Àrea de Recerca Històrica, Andorre, 2001, 367-380.

Le Carlier C., Leroy M., Merluzzo P., 2007. L'apport de l'analyse morphologique, microscopique et chimique des scories en forme de culot à la restitution des activités de forge, revue d'archéométrie, $31: 23-35$.

Lefort A., Marcigny C., 2009. La probable agglomération portuaire du second âge du Fer d'Urville-Nacqueville. Un état de la documentation. Bulletin de l'AMARAI, $22: 39-81$.

Le Goff E. (dir.), 2002. Les occupations protohistoriques et antiques de la ZAC Object'Ifs sud à Ifs (Calvados). Documents Final de Synthèse, INRAP, SRA DRAC de Basse-Normandie, 4 volumes, $1260 \mathrm{p}+$ annexes.

Lepaumier H. (dir.), 2012. Fleury-sur-Orne, Calvados, Les Mézerettes-ZL7. deux habitats enclos et une nécropole du second âge du Fer du réseau de fermes reconnu au sud-est de l'agglomération caennaise. Rapport Final d'Opération, INRAP, SRA DRAC de Basse-Normandie, 2 volumes, 438 et 432 p.

Lepaumier H. (dir.), 1996. A. 29 sud - site 4 - Quetteville, « Les Heurtries II ». Document Final de Synthèse, AFAN, SRA DRAC de Basse-Normandie, $69 \mathrm{p}$.

Lepaumier H., Besnard-Vauterin C.-C., Chanson K., Zaour N., à paraître. Fonction et statut des habitats enclos de la fin de l'âge du Fer, une question de mobilier? L'exemple du réseau d'établissements du sud-est de l'agglomération caennaise. In V. Carpentier, C. Marcigny (dir.). Des hommes aux champs 2 : approches archéologiques des économies agricoles. Actes de la table ronde, (Caen, 29-30 septembre 2011).

Lepaumier H., Giazzon D., Chanson K. (avec la collaboration de L. Féret, V. Guitton et D. Corde), 2011a. Orval, "Les Pleines » (Manche). Habitats enclos et tombe à char en Cotentin. In P. Barral, B. Dedet, F. Delrieu, P. Giraud, I. Le Goff, S. Mario, A. Villard-Le Tiec. Lâge du Fer en Basse-Normandie. Gestes funéraires en Gaule au second âge du Fer. Actes du XXXIII colloque international de l'Association Française pour l'Étude de l'âge du Fer (Caen 2009). Annales Littéraires de l'Université de Franche-Comté, Presses Universitaires de Franche-Comté, 883, série " environnement, société et archéologie », 14, vol. 1, 315-333.

Lepaumier H., Vauterin C.-C., Le Goff E., Villaregut J., 2011b. Un réseau de fermes en périphérie caennaise. In P. Barral, B. Dedet, F. Delrieu, P. Giraud, I. Le Goff, S. Mario, 
A. Villard-Le Tiec. Lâge du Fer en Basse-Normandie. Gestes funéraires en Gaule au second âge du Fer. Actes du XXXIII ${ }^{e}$ colloque international de l'Association Française pour l'Étude de l'âge du Fer (Caen 2009). Annales Littéraires de l'Université de Franche-Comté, Presses Universitaires de Franche-Comté, 883, série « environnement, société et archéologie ", 14, vol. 1, 139-158.

Malrain F., Blancquaert G., Lorho T., 2009. Un enclos = une ferme?. Habitats et paysages ruraux en Gaule et regards sur d'autres régions $d u$ monde celtique. Actes du XXXI colloque international de l'Association Française pour l'Étude de l'âge du Fer (Chauvigny [2007]). Mémoire XXXV de l'Association des Publications Chauvinoises, 25-43.

Mangin M., Fluzin P., 2006. La métallurgie du fer chez les Mandubiens et les Lingons (Haut-Auxois et région des sources de la Seine, Côte d'Or). Un artisanat de qualité. In Artisanats, sociétés et civilisations : hommage à J.-P. Thouvenot. Actes du colloque (Sens 2003), Revue Archéologique de l'Est, supplément $\mathrm{n}^{\circ} 24,523-538$.

Mangin M., Fluzin P., Courtadon J.-L., Fontaine M.-J., 2000. Forgerons et paysans des campagnes d'Alésia (Haut-Auxois, Côtes d'Or, ier siècle av.-viiie siècle apr. J.-C. CNRS édition, Paris, CRA, monographie 22, 508 p.

Marcigny C., Lepaumier H., Carpentier V., ClémentSauleau S., Matterne-Zeck V., Gaumé É., Ghesquière E., Giazzon D., 2004. Un établissement agricole à caractère " aristocratique " du second âge du Fer à Saint-Martin-desEntrées (Calvados). Revue Archéologique de l'Ouest, 21 : 63-94.

Marcigny C., Ghesquière E. (dir.), 2003. L'île de Tatihou (Manche) à l'âge du Bronze. Habitats et occupation du sol. Document d'Archéologie Française, 96, 185 p.

MÉnez Y., 1996. Une ferme de l'Armorique gauloise. Le Boisanne à Plouër-sur-Rance (Côtes d'Armor). Editions des Sciences de l'Homme, Paris, Document d'Archéologie Française, 58, 267 p.

Pellecuer C., 1998. Le travail du fer dans la villa des Près-Bas à Loupian (Hérault). In Feugère M., Serneels V. (dir.). Recherches sur l'économie du fer en Méditerranée nord-occidentale, coll.
"Monographie Instrumentum », 4, éditions Monique Mergoil, Montagnac : 166-174.

San Juan G., Méniel P., Matterne-Zeck V., Savary X., JARDEL K., 1999. L'occupation gauloise au nord-ouest de Caen. L'évaluation du plateau de Thaon (Calvados). Revue Archéologique de l'Ouest, 16 : 131-194.

Serneels V., 1998. La chaîne opératoire de la sidérurgie du fer. In M. Feugère, V. Serneels (dir.). Recherches sur l'économie du fer en Méditerranée nord-occidentale. Collection Monographie Instrumentum, 4, éditions Monique Mergoil, Montagnac, $7-44$.

Serneels V., 1993. Archéométrie des scories de fer. Recherches sur la sidérurgie ancienne en Suisse occidentale. Cahiers d'Archéologie Romande, 61, Lausanne, 240 p.

Vauterin C.-C., Chanson K., Féret L., Le Forestier S., Zaour N., 2011. La culture matérielle de l'âge du Fer : un outil de réflexion sur les sites d'habitats de Basse-Normandie. In P. Barral, B. Dedet, F. Delrieu, P. Giraud, I. Le Goff, S. Mario, A. Villard-Le Tiec. L'âge du Fer en Basse-Normandie. Gestes funéraires en Gaule au second âge du Fer. Actes du XXXIII colloque international de l'Association Française pour l'Étude de l'âge du Fer (Caen 2009). Annales Littéraires de l'Université de Franche-Comté, Presses Universitaires de Franche-Comté, 883, série « environnement, société et archéologie ", 14, vol 1, 203-229.

ZAOUR N., en cours. La métallurgie du fer en Normandie et ses marges de l'âge du Fer au Moyen-Âge : approches interdisciplinaires; archéologique et archéométrique. Thèse de doctorat sous la direction de Ph. Fluzin, UTBM, université de FrancheComté, École Doctorale «Langages, espaces, temps, sociétés ».

ZAour N., 2009a. Les activités métallurgiques en Basse Normandie, la forge de Marcei, Le Maréchal (61). In P. Giraud (dir.). Gaulois sous les Pommiers, catalogue d'exposition, Caen, CG14, 72-74.

Zaour N., 2009b. Les résidus de forge dans En plaine de Caen. In C.-C. Besnard-Vauterin (dir.). Une campagne gauloise et antique, l'occupation du site de l'Étoile à Mondeville. Presses Universitaires de Rennes, coll. "Archéologie et Culture », 121-122. 